\title{
GEOMETRY OF POLYSYMBOLS
}

\author{
MASANORI MORISHITA AND YuJI TERAshima
}

\begin{abstract}
We introduce a multiple generalization of the tame symbol, called polysymbols, associated to meromorphic functions on a Riemann surface as the Massey products in Deligne cohomology, and also give a geometric construction of polysymbols using Chen's iterated integrals. We then deduce some basic properties of polysymbols using our holonomy formula, and show that trivializations of polysymbols give variations of mixed Hodge structure.
\end{abstract}

\section{Introduction}

Let $f$ and $g$ be two meromorphic functions on a closed Riemann surface $\bar{X}$. The tame symbol at $x \in \bar{X}$ defined by

$$
\{f, g\}_{x}=(-1)^{\operatorname{ord}_{x}(f) \operatorname{ord}_{x}(g)} \frac{f^{\operatorname{ord}_{x}(g)}}{g^{\operatorname{ord}_{x}(f)}}(x),
$$

where $\operatorname{ord}_{x}(f)$ stands for the order of $f$ at $x$, is a function-theoretic analog of the Hilbert symbol and plays important roles in class field theory and algebraic $K$-theory. Among many works on the subject, there is a geometric interpretation of the tame symbol $\{f, g\}_{x}$, due to S. Bloch $([\mathrm{Bl}])$ and P. Deligne ([Dl]), as the holonomy of a line bundle with holomorphic connection $\langle f, g\rangle$ over $X=\bar{X} \backslash(S(f) \cup S(g))$ which is given by the cup product $f \cup g$ in the Deligne cohomology $H_{D}^{2}(X, \mathbb{Z}(2))$, where $S(f)$ denotes the set of zeros and poles of $f$. We note that this construction may be regarded as a complex analytic analogue of the linking number of two knots (cf. [Mo]).

The purpose of this paper is to generalize the construction of Bloch and Deligne and introduce a multiple generalization $\left\{f_{1}, \ldots, f_{n}\right\}_{x}$ of the tame symbol associated to meromorphic functions $f_{1}, \ldots, f_{n}$ on $\bar{X}$, following after Milnor-Massey's construction of higher order linking numbers of a link $([\mathrm{Ms}],[\mathrm{Tu}])$. In fact, associated to meromorphic functions $f_{1}, \ldots, f_{n}$ on $\bar{X}$, we introduce the set of isomorphism classes of line bundles with holomorphic connection $\left\langle f_{1}, \ldots, f_{n}\right\rangle$, called a polysymbol, over $X=\bar{X} \backslash \cup_{i=1}^{n} S\left(f_{i}\right)$ as the Massey product in the Deligne cohomology $H_{D}^{2}(X, \mathbb{Z}(n))$, and then define $\left\{f_{1}, \ldots, f_{n}\right\}_{x}$, polysymbol at $x$, by the map $\left\langle f_{1}, \ldots, f_{n}\right\rangle \rightarrow \mathbb{C}$ sending each isomorphism class in $\left\langle f_{1}, \ldots, f_{n}\right\rangle$ to the holonomy of the associated line bundle with connection along a loop encircling $x$. Our theorem (cf. Theorem 2.5 below) then gives an explicit formula for this holonomy in terms of Chen's iterated integrals $([\mathrm{C} 2])$. We also give a geometric construction of the polysymbol $\left\langle f_{1}, \ldots, f_{n}\right\rangle$ which may be regarded as a natural extension of Bloch-Ramakrishnan's ([Bl],[R]) and Hain's $([\mathrm{H}])$.

Received by the editors August 25, 2006.

2000 Mathematics Subject Classification. 11G45, 14F43, 19F15, 55S30, 58A99.

Key words and phrases. polysymbol, Deligne cohomology, Massey product, iterated integral. 
Namely, using the iterated integrals, we define a map associated to $f_{1}, \ldots, f_{n}$ and a defining system $A$ for $\left\langle f_{1}, \ldots, f_{n}\right\rangle$

$$
T\left(f_{1}, \ldots, f_{n}\right)_{A}: X \longrightarrow N(\mathbb{Z}) \backslash N(\mathbb{C}) / C,
$$

where $N(R)$ for a commutative ring $R$ denotes the group of $R$-valued points of the Heisenberg group of degree $n+1$ and $C$ is the center of $N(\mathbb{C})$. The manifold $P:=$ $N(\mathbb{Z}) \backslash N(\mathbb{C})$ has a natural structure of a principal $\mathbb{C}^{\times}$-bundle over $N(\mathbb{Z}) \backslash N(\mathbb{C}) / C$ and carries a standard connection form $\theta$. By comparing the holonomies, the polysymbol $\left\langle f_{1}, \ldots, f_{n}\right\rangle_{A}$ relative to $A$ is interpreted as the isomorphism class of the pull-back of the bundle with connection $(P, \theta)$ under $T\left(f_{1}, \ldots, f_{n}\right)_{A}$. We then show, using our holonomy formula, some basic properties of polysymbols which generalize those of the tame symbol. Finally, we show that trivializations of polysymbols give variations of mixed Hodge structure.

\section{Deligne cohomology and tame symbols}

In this section, for the convenience of readers, we recall the basic materials on Deligne cohomology which will be used in the sequel. References are $[\mathrm{Br}]$ and $[\mathrm{EV}]$.

Let $X$ be a complex manifold. The Deligne cohomology, denoted by $H_{D}^{*}(X, \mathbb{Z}(p))$ for an integer $p \geq 1$, is by definition the hypercohomology of the Deligne complex $\mathbb{Z}(p)_{D}$ defined by the complex of sheaves on $X$

$$
\mathbb{Z}(p):=(2 \pi \sqrt{-1})^{p} \mathbb{Z} \rightarrow \mathcal{O}_{X} \stackrel{d}{\rightarrow} \Omega_{X}^{1} \stackrel{d}{\rightarrow} \cdots \stackrel{d}{\rightarrow} \Omega_{X}^{p-1}
$$

where $\mathbb{Z}(p)$ is in degree 0 and $\mathcal{O}_{X}$ and $\Omega_{X}^{k}(1 \leq k \leq p-1)$ denote the sheaf of holomorphic functions and of holomorphic $k$-forms on $X$ respectively. The map $\mathcal{O}_{X} \rightarrow$ $\mathcal{O}_{X}^{\times}$defined by $x \mapsto \exp \left(\frac{1}{(2 \pi \sqrt{-1})^{p-1}} x\right)$ and the multiplication on $\Omega_{X}^{k}$ by $\frac{1}{(2 \pi \sqrt{-1})^{p-1}}$ induce a quasi-isomorphism

$$
\mathbb{Z}(p)_{D} \simeq\left(\mathcal{O}_{X}^{\times} \stackrel{d \log }{\rightarrow} \Omega_{X}^{1} \rightarrow \cdots \rightarrow \Omega_{X}^{p-1}\right)[-1]
$$

and hence we have the isomorphisms

$$
H_{D}^{q}(X, \mathbb{Z}(p)) \simeq \mathbb{H}^{q-1}\left(X, \mathcal{O}_{X}^{\times} \stackrel{d \log }{\rightarrow} \Omega_{X}^{1} \rightarrow \cdots \rightarrow \Omega_{X}^{p-1}\right), \quad q \geq 1 .
$$

For low values of $p$ and $q$, the isomorphisms above are given as follows.

1.1. $p=q=1$ : An element of $H_{D}^{1}(X, \mathbb{Z}(1))$ is repsesented by a Čech 1 -cocycle $\alpha$ for a suitable open cover $\left\{U_{a}\right\}$ of $X$

$$
\alpha=\left(q_{a b}, F_{a}\right) \in C^{1}(\mathbb{Z}(1)) \oplus C^{0}\left(\mathcal{O}_{X}\right), \quad q_{a b}=\delta F_{a}
$$

where $\delta$ stands for the boundary map for Čech cocycles with respect to open covers. Then we associate to $\alpha$ the function $f \in H^{0}\left(X, \mathcal{O}_{X}^{\times}\right)$so that $F_{a}$ is a single branch of $\log f$ restricted on $U_{a}$. This yields the isomorphism

$$
H_{D}^{1}(X, \mathbb{Z}(1)) \simeq H^{0}\left(X, \mathcal{O}_{X}^{\times}\right)
$$

In the following, for $f \in H^{0}\left(X, \mathcal{O}_{X}^{\times}\right)$, we denote by $\log _{a_{1} \ldots a_{r}}(f)$ for a single branch of $\log f$ restricted on $U_{a_{1} \ldots a_{r}}:=U_{a_{1}} \cap \cdots \cap U_{a_{r}}$. 
1.2. $p=q=2:$ An element of $H_{D}^{2}(X, \mathbb{Z}(2))$ is represented by a Čech 2-cocycle

$$
\beta=\left(q_{a b c}, \log _{a b} f, \Omega_{a}\right) \in C^{2}(\mathbb{Z}(2)) \oplus C^{1}\left(\mathcal{O}_{X}\right) \oplus C^{0}\left(\Omega_{X}^{1}\right)
$$

which is subject to the relations $q_{a b c}=\delta \log _{a b} f, d \log _{a b} f=\delta \Omega_{a}$. We then associate to $\beta$ the Čech 1-cocycle with value in the complex $\mathcal{O}_{X}^{\times} \stackrel{d \log }{\longrightarrow} \Omega_{X}^{1}$

$$
\left(\xi_{a b}, \omega_{a}\right) \in C^{1}\left(\mathcal{O}_{X}^{\times}\right) \oplus C^{0}\left(\Omega_{X}^{1}\right), \xi_{a b}=\exp \left(\frac{1}{2 \pi \sqrt{-1}} \log _{a b} f\right), \omega_{a}=\frac{1}{2 \pi \sqrt{-1}} \Omega_{a}
$$

with $\delta \xi_{a b}=1, d \log \xi_{a b}=\delta \omega_{a}$. This gives the isomorphism

$$
H_{D}^{2}(X, \mathbb{Z}(2)) \simeq \mathbb{H}^{1}\left(X, \mathcal{O}_{X}^{\times} \stackrel{d \log }{\longrightarrow} \Omega_{X}^{1}\right) .
$$

1.3. The hypercohomology $\mathbb{H}^{1}\left(X, \mathcal{O}_{X}^{\times} \stackrel{d \log }{\longrightarrow} \Omega_{X}^{1}\right)$ is interpreted as the group of isomorphism classes of line bundles over $X$ with holomorphic connection, where the group structure of the latter is induced by the tensor product of bundles with connection. Suppose we are given such a pair $(L, \nabla)$. As usual, we identify $L$ with the associated principal $\mathbb{C}^{\times}$-bundle $L^{+}$. Take a suitable open cover $\left\{U_{a}\right\}$ of $X$ such that $L^{+}$has a section $s_{a}$ over $U_{a}$. Then the pair $\left(\xi_{a b}, \omega_{a}\right)$ defined by $\xi_{a b}:=\frac{s_{b}}{s_{a}}$ and $\omega_{a}:=\frac{\nabla\left(s_{a}\right)}{s_{a}}$ gives a Cech 1-cocycle with value in the complex $\mathcal{O}_{X}^{\times} \stackrel{d \log }{\rightarrow} \Omega_{X}^{1}$ by the relation $d \log \xi_{a b}=\delta \omega_{a}$. Conversely, we easily see that any such cocycle comes from the pair $(L, \nabla)$. This association preserves the group structures. We also note that when $X$ is a Riemann surface, $H_{D}^{2}(X, \mathbb{Z}(2))$ is isomorphic to $H^{1}\left(X, \mathbb{C}^{\times}\right)$, the group of isomorphism classes of smooth flat line bundles on $X$.

1.4. A crucial property of Deligne cohomology for our purpose is that it is equipped with a cup product on Deligne complexes

$$
\mathbb{Z}(p)_{D} \otimes \mathbb{Z}\left(p^{\prime}\right)_{D} \rightarrow \mathbb{Z}\left(p+p^{\prime}\right)_{D}
$$

given by

$$
x \cup y= \begin{cases}x \cdot y & \operatorname{deg}(x)=0, \\ x \wedge d y & \operatorname{deg}(x) \geq 0, \operatorname{deg}(y)=p^{\prime}, \\ 0, & \text { otherwise. }\end{cases}
$$

The cup product is anti-commutative up to homotopy and so we have $\alpha \cup \beta=$ $(-1)^{q r} \beta \cup \alpha$ for $\alpha \in H_{D}^{q}(X, \mathbb{Z}(p)), \beta \in H_{D}^{r}\left(X, \mathbb{Z}\left(p^{\prime}\right)\right)$.

1.5. As an application of the cup product, one can describe the tame symbol $\{f, g\}_{x}$ in terms of Deligne cohomology. Suppose that $f$ and $g$ are meromorphic functions on a closed Riemann surface $\bar{X}$. Set $X=\bar{X} \backslash(S(f) \cup S(g))$ so that $f, g \in H^{0}\left(X, \mathcal{O}_{X}^{\times}\right)$, where $S(f)$ denotes the set of zeros and poles of $f$. By 1.1, $f$ and $g$ are represented by the 1-cocycles

$$
\left(q_{a b}, \log _{a} f\right) \text { and }\left(q_{a b}^{\prime}, \log _{a} g\right)
$$

respectively with $q_{a b}=\delta \log _{a} f, q_{a b}^{\prime}=\delta \log _{a} g$. By 1.4, the cup product $f \cup g$ is represented by the 2 -cocycle

$$
\left(q_{a b} q_{b c}^{\prime}, q_{a b} \log _{b} g, \log _{a} f \frac{d g}{g}\right)
$$


which corresponds to the 1 -cocycle with value in the complex $\mathcal{O}_{X}^{\times} \stackrel{d \log }{\rightarrow} \Omega_{X}^{1}$ via (1.2)

$$
\left(g^{\frac{q_{a b}}{2 \pi \sqrt{-1}}}, \frac{1}{2 \pi \sqrt{-1}} \log _{a} f \frac{d g}{g}\right) .
$$

We write $\langle f, g\rangle$ for the corresponding isomorphism class of line bundles with holomorphic connection on $X$ by 1.3. Noting that $\frac{q_{a b}}{2 \pi \sqrt{-1}}=-\left(\log _{a} f-\log _{b} f\right)$, the holonomy of $\langle f, g\rangle$ along a loop $l$ with base point $x_{0}$ in $X$ is given by

$$
\exp \frac{1}{2 \pi \sqrt{-1}}\left(\int_{l} \log f \frac{d g}{g}-\log g\left(x_{0}\right) \int_{l} \frac{d f}{f}\right)
$$

which is precisely the tame symbol $\{f, g\}_{x}$ at $x \in X$ in (0.1) by Cauchy's theorem when $l$ is a small loop encircling $x$.

\section{Massey products and polysymbols}

In this section, generalizing the construction in 1.5, we introduce polysymbols as Massey products in Deligne cohomology, and compute their holonomies explicitly in terms of Chen's iterated integrals. For Massey products and iterated integrals in general contexts, we refer to $[\mathrm{Kr}],[\mathrm{My}]$ and $[\mathrm{C} 2]$.

Let $\bar{X}$ be a closed Riemann surface. Let $f_{1}, \cdots, f_{n}$ be meromorphic functions on $\bar{X}$ and let $S\left(f_{i}\right)$ be the set of zeros and poles of $f$. Set $X=\bar{X} \backslash \cup_{i=1}^{n} S\left(f_{i}\right)$ so that $f_{i} \in H^{0}\left(X, \mathcal{O}_{X}^{\times}\right)=H_{D}^{1}(X, \mathbb{Z}(1))$ by 1.1 .

In the following, we omit the subscripts $a, a b, \cdots$ in the Čech cochains, which stand for open subsets $U_{a}, U_{a b}, \cdots$, for the sake of simplicity.

Definition 2.1. The Massey product $\left\langle f_{1}, \ldots, f_{n}\right\rangle$ is said to be defined if there is an array $A$ of Čech 1-cochains

$$
A=\left\{\alpha_{i_{1} \cdot i_{k}}=\left(q_{i_{1} \cdot i_{k}}, \log f_{i_{1} \cdot i_{k}}\right) \in C^{1}(\mathbb{Z}(k)) \oplus C^{0}\left(\mathcal{O}_{X}\right) \mid i_{p+1}=i_{p}+1(\forall p)\right\}_{1 \leq k \leq n-1}
$$

such that

(2.1.1) $\alpha_{i}=\left(q_{i}, \log f_{i}\right)$ is a 1-cocycle representing $f_{i}$ for $1 \leq i \leq n$,

(2.1.2) $D \alpha_{i_{1} \cdot i_{k}}=\alpha_{i_{1} \cdot i_{k}-1} \cup \alpha_{i_{k}}+\alpha_{i_{1} \cdot i_{k}-2} \cup \alpha_{i_{k}-1 i_{k}}+\cdots+\alpha_{i_{1}} \cup \alpha_{i_{2} \cdot i_{k}}$ for $1 \leq k \leq$ $n-1, i_{p+1}=i_{p}+1$ where $D$ stands for the boundary operator of Deligne cohomology.

An array $A$ is called a defining system for $\left\langle f_{1}, \ldots, f_{n}\right\rangle$. The value of the Massey product relative to a defining system $A$, denoted by $\left\langle f_{1}, \ldots, f_{n}\right\rangle_{A}$, is then defined to be the cohomology class of $H_{D}^{2}(X, \mathbb{Z}(n))$ represented by the 2-cocycle

$$
c(A):=\alpha_{1 \cdot n-1} \cup \alpha_{n}+\alpha_{1 \cdot n-2} \cup \alpha_{n-1 n}+\cdots+\alpha_{1} \cup \alpha_{2 \cdot n n} .
$$

The Massey product $\left\langle f_{1}, \ldots, f_{n}\right\rangle$ itself is usually taken to be the subset of $H_{D}^{2}(X, \mathbb{Z}(n))$ consisting of $\left\langle f_{1}, \ldots, f_{n}\right\rangle_{A}$ for some defining system $A$.

Since $\operatorname{dim} X=1$, the multiplication by $\frac{1}{(2 \pi \sqrt{-1})^{n-2}}$ induces the isomorphism

$$
H_{D}^{2}(X, \mathbb{Z}(n)) \simeq H_{D}^{2}(X, \mathbb{Z}(2))
$$


and hence each $\left\langle f_{1}, \ldots, f_{n}\right\rangle_{A}$ is identified with an isomorphism class of line bundles over $X$ with holomorphic connection by 1.3 via $(2.2)$. We also note that a line bundle over $X$ with holomorphic connection is flat, namely its curvature is zero, since $\operatorname{dim} X=1$.

Definition 2.3. We call $\left\langle f_{1}, \ldots, f_{n}\right\rangle_{A}$ and the associated isomorphism class of line bundles over $X$ with holomorphic connection a polysymbol relative to $A$, and call $\left\langle f_{1}, \ldots, f_{n}\right\rangle$ simply the polysymbol for meromorphic functions $f_{1}, \ldots, f_{n}$.

For $x \in \bar{X}$, let $H_{l_{x}}\left(\left\langle f_{1}, \ldots, f_{n}\right\rangle_{A}\right)$ denote the holonomy of $\left\langle f_{1}, \ldots, f_{n}\right\rangle_{A}$ along a small loop $l_{x}$ encircling $x$ and we define the polysymbol at $x$, denoted by $\left\{f_{1}, \ldots, f_{n}\right\}_{x}$, by the map

$$
\left\{f_{1}, \ldots, f_{n}\right\}_{x}:\left\langle f_{1}, \ldots, f_{n}\right\rangle \rightarrow \mathbb{C} ;\left\langle f_{1}, \ldots, f_{n}\right\rangle_{A} \mapsto H_{l_{x}}\left(\left\langle f_{1}, \ldots, f_{n}\right\rangle_{A}\right)
$$

It is easily seen by Stokes' theorem and the flatness of $\left\langle f_{1}, \ldots, f_{n}\right\rangle_{A}$ that the map $\left\{f_{1}, \ldots, f_{n}\right\}_{x}$ is well-defined, namely depends only on $f_{1}, \ldots, f_{n}$ and $x$.

Remark 2.4. (1) For $n=2,\left\langle f_{1}, f_{2}\right\rangle$ consists of a single class $f_{1} \cup f_{2}$ and so the map $\left\{f_{1}, f_{2}\right\}_{x}$ in Definition 2.3 is identified with the classical tame symbol by 1.5 .

(2) Suppose that all $k$-th order polysymbols $\left\{g_{1}, \ldots, g_{k}\right\}_{x}$ at $x$ are 1 for any $k<n$ and all meromorphic functions $g_{1}, \ldots, g_{k}$. It implies that all $k$-th order polysymbols $\left\langle g_{1}, \ldots, g_{k}\right\rangle$ restricted to a neighborhood of $x$ are trivial for any $k<n$. Then, by the well known property of Massey products $([\mathrm{Kr}],[\mathrm{My}])$, the $n$-th polysymbol $\left\langle f_{1}, \ldots, f_{n}\right\rangle$ restricted on a neighborhood of $x$ consists of a single cohomology class and hence $\left\{f_{1}, \ldots, f_{n}\right\}_{x} \in \mathbb{C}$ is numerically defined.

Now, we compute the holonomy of a polysymbol $\left\langle f_{1}, \ldots, f_{n}\right\rangle_{A}$ relative to a defining system $A$ in terms of Chen's iterated integrals ([C2]). Recall that for 1 -forms $w_{1}, \ldots, w_{n}$ on $X$ and a path $\gamma:[0,1] \rightarrow X$ with $\gamma^{*} w_{i}=F_{i}(t) d t(1 \leq i \leq n)$, the iterated integral $\int_{\gamma} w_{1} \cdots w_{n}$ is defined by

$$
\int_{\gamma} w_{1} \cdots w_{n}:=\int_{0 \leq t_{1}<\cdots<t_{n} \leq 1} F_{1}\left(t_{1}\right) \cdots F_{n}\left(t_{n}\right) d t_{1} \cdots d t_{n} .
$$

Theorem 2.5. For a loop $l$ with base point $x_{0}$ in $X$, the holonomy of the polysymbol $\left\langle f_{1}, \ldots, f_{n}\right\rangle_{A}$ relative to a defining system $A$ (Definition 2.1) along $l$, denoted by $H_{l}\left(\left\langle f_{1}, \ldots, f_{n}\right\rangle_{A}\right)$, is given by

$$
H_{l}\left(\left\langle f_{1}, \ldots, f_{n}\right\rangle_{A}\right)=\exp \left(\frac{1}{(2 \pi \sqrt{-1})^{n-1}} M_{12 \cdots n}(l)\right)
$$

where $M_{12 \ldots n}(l)$ is given by 


$$
\begin{aligned}
& \int_{l} \frac{d f_{1}}{f_{1}} \ldots \frac{d f_{n}}{f_{n}}+\log f_{1}\left(x_{0}\right) \int_{l} \frac{d f_{2}}{f_{2}} \ldots \frac{d f_{n}}{f_{n}}+\cdots+\log f_{1 \cdots n-1}\left(x_{0}\right) \int_{l} \frac{d f_{n}}{f_{n}} \\
& +\sum_{\substack{i+j+k=n \\
i \geq 0, j \geq 1, k \geq 1}} \sum_{k_{1}+\cdots+k_{p}=k}(-1)^{p} \log f_{1 \cdot i}\left(x_{0}\right) \int_{l} \frac{d f_{i+1}}{f_{i+1}} \ldots \frac{d f_{i+j}}{f_{i+j}} \log f_{i+j+1 \cdot \cdot i+j+k_{1}}\left(x_{0}\right) \\
& \quad \times \log f_{i+j+k_{1}+1 \cdot i+j+k_{1}+k_{2}}\left(x_{0}\right) \ldots \log f_{i+j+k_{1}+\cdot \cdot+k_{p-1}+1 \cdot n}\left(x_{0}\right)
\end{aligned}
$$

For example, we have

$$
\begin{aligned}
M_{12}(l)= & \int_{l} \frac{d f_{1}}{f_{1}} \frac{d f_{2}}{f_{2}}+\log f_{1}\left(x_{0}\right) \int_{l} \frac{d f_{2}}{f_{2}}-\log f_{2}\left(x_{0}\right) \int_{l} \frac{d f_{1}}{f_{1}} \\
M_{123}(l)= & \int_{l} \frac{d f_{1}}{f_{1}} \frac{d f_{2}}{f_{2}} \frac{d f_{3}}{f_{3}}+\log f_{1}\left(x_{0}\right) \int_{l} \frac{d f_{2}}{f_{2}} \frac{d f_{3}}{f_{3}}+\log f_{12}\left(x_{0}\right) \int_{l} \frac{d f_{3}}{f_{3}} \\
& -\int_{l} \frac{d f_{1}}{f_{1}} \log f_{23}\left(x_{0}\right)-\int_{l} \frac{d f_{1}}{f_{1}} \frac{d f_{2}}{f_{2}} \log f_{3}\left(x_{0}\right)-\log f_{1}\left(x_{0}\right) \int_{l} \frac{d f_{2}}{f_{2}} \log f_{3}\left(x_{0}\right) \\
& +\int_{l} \frac{d f_{1}}{f_{1}} \log f_{2}\left(x_{0}\right) \log f_{3}\left(x_{0}\right)
\end{aligned}
$$

Proof. First, note that the condition (2.1.2) is expressed in terms of cocycles by

$$
\begin{aligned}
& \left(\delta q_{i_{1} \cdot i_{k}},-q_{i_{1} \cdot i_{k}}+\delta \log f_{i_{1} \cdot i_{k}}, \frac{d f_{i_{1} \cdot i_{k}}}{f_{i_{1} \cdot i_{k}}}\right) \\
& =\left(\begin{array}{l}
q_{i_{1} \cdot i_{k}-1} q_{i_{k}}+q_{i_{1} \cdot i_{k}-2} q_{i_{k}-1 i_{k}}+\cdot \cdot+q_{i_{1}} q_{i_{2} \cdot \cdot i_{k}}, \\
q_{i_{1} \cdot i_{k}-1} \log f_{i_{k}}+q_{i_{1} \cdot i_{k}-2} \log f_{i_{k}-1 i_{k}}+\cdot \cdot+q_{i_{1}} \log f_{i_{2} \cdot i_{k}}, \log f_{i_{1} \cdot i_{k}-1} \frac{d f_{i_{k}}}{f_{i_{k}}}
\end{array}\right)
\end{aligned}
$$

In particular, we have

$$
q_{1 \cdot k}=\delta \log f_{1 \cdot k}-\left(q_{1 \cdot * k-1} \log f_{k}+q_{1 \cdot * k-2} \log f_{k-1 k}+\cdots+q_{1} \log f_{2 \cdot k}\right) .
$$

The cocycle $c(A)$ is expressed by

(2.8) $c(A)=\left(*, q_{1 \cdot n-1} \log f_{n}+q_{1 \cdot \cdot n-2} \log f_{n-1 n}+\cdots+q_{1} \log f_{2 \cdot n n}, \log f_{1 \cdot n-1} \frac{d f_{n}}{f_{n}}\right)$.

By (2.7) and (2.8), we have

$$
c(A)=\left(\begin{array}{c}
*, \sum_{\substack{l+k=n \\
l \geq 1, k \geq 1}} \sum_{k_{1}+\cdots+k_{p}=k}(-1)^{p-1} \delta \log f_{1 \cdots l} \log f_{l+1 \cdot \cdot l+k_{1}} \\
\times \log f_{l+k_{1}+1 \cdot l+k_{1}+k_{2}} \ldots \log f_{l+k_{1}+\cdot \cdot+k_{p-1}+1 \cdot n}, \log f_{1 \cdot n-1} \frac{d f_{n}}{f_{n}}
\end{array}\right) .
$$

Using the relation $\frac{d f_{i_{1} \cdot i_{k}}}{f_{i_{1} \cdot \cdots i_{k}}}=\log f_{i_{1} \cdot \cdot i_{k}-1} \frac{d f_{i_{k}}}{f_{i_{k}}}$ from (2.6), the contribution of the term 
$\log f_{1 \cdot \cdot n} \frac{d f_{n}}{f_{n}}$ to the holonomy is exp of

$$
\begin{aligned}
& \int_{l} \log f_{1 \cdot \cdot n-1} \frac{d f_{n}}{f_{n}} \\
& =\log f_{1 \cdot \cdot n-1}\left(x_{0}\right) \int_{l} \frac{d f_{n}}{f_{n}}+\int_{l} \frac{d f_{1 \cdot \cdot n-1}}{f_{1 \cdot \cdot n-1}} \frac{d f_{n}}{f_{n}} \\
& =\log f_{1 \cdot \cdot n-1}\left(x_{0}\right) \int_{l} \frac{d f_{n}}{f_{n}}+\int_{l}\left(\log f_{1 \cdot \cdot n-2} \frac{d f_{n-1}}{f_{n-1}}\right) \frac{d f_{n}}{f_{n}} \\
& =\log f_{1 \cdot \cdot n-1}\left(x_{0}\right) \int_{l} \frac{d f_{n}}{f_{n}}+\log f_{1 \cdot \cdot n-2}\left(x_{0}\right) \int_{l} \frac{d f_{n-1}}{f_{n-1}} \frac{d f_{n}}{f_{n}}+\int_{l} \frac{d f_{1 \cdot n-2}}{f_{1 \cdot n-2}} \frac{d f_{n-1}}{f_{n-1}} \frac{d f_{n}}{f_{n}} \\
& \quad \ldots \\
& =\log f_{1 \cdot \cdot n-1}\left(x_{0}\right) \int_{l} \frac{d f_{n}}{f_{n}}+\log f_{1 \cdot \cdot n-2}\left(x_{0}\right) \int_{l} \frac{d f_{n-1}}{f_{n-1}} \frac{d f_{n}}{f_{n}}+\cdots+\log f_{1}\left(x_{0}\right) \int_{l} \frac{d f_{2}}{f_{2}} \ldots \frac{d f_{n}}{f_{n}} \\
& \quad+\int_{l} \frac{d f_{1}}{f_{1}} \ldots \frac{d f_{n}}{f_{n}} .
\end{aligned}
$$

The contribution of the term

$$
\left(-\delta \log f_{1 \cdot \cdot l}\right) \log f_{l+1 \cdot \cdot l+k_{1}} \log f_{l+k_{1}+1 \cdot \cdot l+k_{1}+k_{2}} \ldots \log f_{l+k_{1}+\cdot \cdot+k_{p-1}+1 \cdot \cdot n}
$$

to the holonomy is exp of

$$
\begin{aligned}
\left(\int_{l} \frac{d f_{1 \cdot \cdot l}}{f_{1 \cdot \cdot l}}\right) \log f_{l+1 \cdot \cdot l+k_{1}}\left(x_{0}\right) \log f_{l+k_{1}+1 \cdot \cdot l+k_{1}+k_{2}}\left(x_{0}\right) \ldots \log f_{l+k_{1}+\cdot \cdot+k_{p-1}+1 \cdot \cdot n}\left(x_{0}\right) \\
=\left(\int_{l} \log f_{1 \cdot l-1} \frac{d f_{l}}{f_{l}}\right) \log f_{l+1 \cdot \cdot l+k_{1}}\left(x_{0}\right) \\
\quad \times \log f_{l+k_{1}+1 \cdot \cdot+k_{1}+k_{2}}\left(x_{0}\right) \ldots \log f_{l+k_{1}+\cdot \cdot+k_{p-1}+1 \cdot n}\left(x_{0}\right) \\
=\left(\log f_{1 \cdot \cdot l-1}\left(x_{0}\right) \int_{l} \frac{d f_{l}}{f_{l}}+\log f_{1 \cdot \cdot l-2}\left(x_{0}\right) \int_{l} \frac{d f_{l-1}}{f_{l-1}} \frac{d f_{l}}{f_{l}}+\cdots+\log f_{1}\left(x_{0}\right) \int_{l} \frac{d f_{2}}{f_{2}} \ldots \frac{d f_{l}}{f_{l}}\right. \\
\left.\quad+\int_{l} \frac{d f_{1}}{f_{1}} \ldots \frac{d f_{l}}{f_{l}}\right) \log f_{l+1 \cdot \cdot+k_{1}}\left(x_{0}\right) \\
\quad \times \log f_{l+k_{1}+1 \cdot \cdot l+k_{1}+k_{2}}\left(x_{0}\right) \ldots \log f_{l+k_{1}+\cdot \cdot+k_{p-1}+1 \cdot \cdot n}\left(x_{0}\right) \\
=\sum_{\substack{i+j=l \\
i \geq 0, j \geq 1}} \log f_{1 \cdot \cdot i}\left(x_{0}\right) \int_{l} \frac{d f_{i+1}}{f_{i+1}} \ldots \frac{d f_{i+j}}{f_{i+j}} \log f_{i+j+1 \cdot \cdot i+j+k_{1}}\left(x_{0}\right) \\
\quad \times \log f_{i+j+k_{1}+1 \cdot \cdot i+j+k_{1}+k_{2}}\left(x_{0}\right) \ldots \log f_{i+j+k_{1}+\cdot \cdot+k_{p-1}+1 \cdot n}\left(x_{0}\right) .
\end{aligned}
$$

Since $\left\langle f_{1}, \ldots, f_{n}\right\rangle_{A}$ corresponds to the class $\left[\frac{1}{(2 \pi \sqrt{-1})^{n-1}} c(A)\right] \in H^{2}(X, \mathbb{Z}(2))$, getting all these together, we obtain the desired formula.

\section{Geometirc construction of polysymbols}

In this section, we give a geometric construction of the isomorphism class of line bundles with holomorphic connection represented by the polysymbol $\left\langle f_{1}, \cdots, f_{n}\right\rangle_{A}$ relative to a defining system $A$ introduced in Section 2. Our method may be regarded as a natural generalization of Bloch-Ramakrishnan $([\mathrm{Bl}],[\mathrm{R}])$ and Hain's $([\mathrm{H}])$. We 
keep the same notations as in Section 2.

Let $N=N_{n+1}$ be the Heisenberg group of degree $n+1$ so that the set of $R$-valued points of $N$ for any commutative ring $R$ is given by

$$
N(R):=\left\{\left(\begin{array}{cccccc}
1 & x_{1} & x_{12} & \ldots & x_{12 \ldots n-1} & x_{12 \ldots n} \\
0 & 1 & x_{2} & x_{23} & \ldots & x_{23 \ldots n} \\
\vdots & \ddots & \ddots & \ddots & \ddots & \vdots \\
& & & 1 & x_{n-1} & x_{n-1 n} \\
& & & & 1 & x_{n} \\
0 & \ldots & & & 0 & 1
\end{array}\right) \mid x_{i_{1} \cdot i_{k}} \in R\right\} .
$$

We set

$$
\begin{gathered}
C:=\left\{\left(\begin{array}{cccccc}
1 & 0 & & \cdots & 0 & z \\
0 & 1 & 0 & & \cdots & 0 \\
\vdots & \ddots & \ddots & \ddots & \ddots & \vdots \\
\vdots & & & \ddots & 1 & 0 \\
0 & \ldots & & \cdots & 0 & 1
\end{array}\right) \mid z \in \mathbb{C}\right\}=\text { the center of } N(\mathbb{C}) \simeq \mathbb{C}, \\
B:=N(\mathbb{Z}) \backslash N(\mathbb{C}) / C .
\end{gathered}
$$

We fix base points $x_{0} \in X$ and $N(\mathbb{Z}) A C \in B$ where the matrix $A$ is given by

$$
A:=\left(\begin{array}{cccccc}
1 & a_{1} & a_{12} & \ldots & a_{12 \ldots n-1} & 0 \\
& 1 & a_{2} & a_{23} & \ldots & a_{23 \ldots n} \\
& & \ddots & \ddots & \ddots & \vdots \\
& & & 1 & a_{n-1} & a_{n-1 n} \\
& & & & 1 & a_{n} \\
& & & & & 1
\end{array}\right)
$$

For simplicity, we set

$$
\omega_{i}:=\frac{1}{2 \pi \sqrt{-1}} \frac{d f_{i}}{f_{i}} .
$$

We then define a holomorphic map

$$
T\left(f_{1}, \ldots, f_{n}\right)_{A}: X \longrightarrow B
$$

by

$$
\begin{aligned}
& T\left(f_{1}, \ldots, f_{n}\right)_{A}(x) \\
& :=N(\mathbb{Z}) A\left(\begin{array}{cccccc}
1 & \int_{\gamma_{x}} \omega_{1} & \int_{\gamma_{x}} \omega_{1} \omega_{2} & \ldots & \int_{\gamma_{x}} \omega_{1} \ldots \omega_{n-1} & 0 \\
& 1 & \int_{\gamma_{x}} \omega_{2} & \int_{\gamma_{x}} \omega_{2} \omega_{3} & \ldots & \int_{\gamma_{x}} \omega_{2} \ldots \omega_{n} \\
& & \ddots & \ddots & \ddots & \vdots \\
& & & 1 & \int_{\gamma_{x}} \omega_{n-1} & \int_{\gamma_{x}} \omega_{n-1} \omega_{n} \\
& & & & & \int_{\gamma_{x}} \omega_{n} \\
& & & & & 1
\end{array}\right) C
\end{aligned}
$$


where $\gamma_{x}$ is a path from $x_{0}$ to $x$ in $X$.

For a loop $l$ based at $x_{0}$, we define $m_{i_{1} \ldots i_{k}}^{\prime}(l)$ and $m_{i_{1} \ldots i_{k}}(l) \in \mathbb{C}$ inductively as follows:

$$
\begin{aligned}
m_{i}^{\prime}(l):= & \int_{l} \omega_{i}=: m_{i}(l), \quad m_{i_{1} \ldots i_{k}}^{\prime}(l):=\int_{l} \omega_{i_{1}} \ldots \omega_{i_{k}} \\
m_{i_{1} \ldots i_{k}}(l):= & m_{i_{1} \ldots i_{k}}^{\prime}(l)+a_{i_{1}} m_{i_{2} \ldots i_{k}}^{\prime}(l)+a_{i_{1} i_{2}} m_{i_{3} \ldots i_{k}}^{\prime}(l)+\cdots+a_{i_{1} \ldots i_{k-1}} m_{i_{k}}^{\prime}(l) \\
& \quad-\left(m_{i_{1} \ldots i_{k-1}}(l) a_{i_{k}}+m_{i_{1} \ldots i_{k-2}}(l) a_{i_{k-1} i_{k}}+\cdots+m_{i_{1}}(l) a_{i_{2} \ldots i_{k}}\right) .
\end{aligned}
$$

For example, we have

$$
\begin{aligned}
& m_{12}(l)=m_{12}^{\prime}(l)+a_{1} m_{2}^{\prime}(l)-m_{1}(l) a_{2} \\
& =\int_{l} \omega_{1} \omega_{2}+a_{1} \int_{l} \omega_{2}-\int_{l} \omega_{1} a_{2}, \\
& m_{123}(l)=m_{123}^{\prime}(l)+a_{1} m_{23}^{\prime}(l)+a_{12} m_{3}^{\prime}(l)-\left(m_{1}(l) a_{23}+m_{12}(l) a_{3}\right) \\
& =\int_{l} \omega_{1} \omega_{2} \omega_{3}+a_{1} \int_{l} \omega_{2} \omega_{3}+a_{12} \int_{l} \omega_{3} \\
& -\left(\int_{l} \omega_{1} a_{23}+\int_{l} \omega_{1} \omega_{2} a_{3}+a_{1} \int_{l} \omega_{2} a_{3}-\int_{l} \omega_{1} a_{2} a_{3}\right), \\
& \text {..... } \\
& m_{12 \ldots n}(l)=m_{12 \ldots n}^{\prime}(l)+a_{1} m_{2 \ldots n}^{\prime}(l)+\cdots+a_{1 \ldots n-1} m_{n}^{\prime}(l) \\
& -\left(m_{1 \ldots n-1}(l) a_{n}+m_{1 \ldots n-2}(l) a_{n-1 n}+\cdots+m_{1}(l) a_{2 \ldots n}\right) \\
& =\int_{l} \omega_{1} \ldots \omega_{n}+a_{1} \int_{l} \omega_{2} \ldots \omega_{n}+\cdots+a_{1 \cdot n-1} \int_{l} \omega_{n} \\
& -\sum_{\substack{i+j+k=n \\
i \geq 0, j \geq 1, k \geq 1}} \sum_{k_{1}+\cdots+k_{p}=k}(-1)^{p-1} a_{1 \cdots i} \int_{l} \omega_{i+1} \ldots \omega_{i+j} \\
& \times a_{i+j+1 \cdot \cdot i+j+k_{1}} a_{i+j+k_{1}+1 \cdot \cdot i+j+k_{1}+k_{2}} \ldots a_{i+j+k_{1}+\cdot \cdot+k_{p-1}+1 \cdot \cdot n} .
\end{aligned}
$$

Lemma 3.2. Under the assumption that

$$
m_{i_{1} \ldots i_{k}}(l) \in \mathbb{Z}\left(1 \leq k \leq n-1, i_{p+1}=i_{p}+1,[l] \in \pi_{1}\left(X, x_{0}\right)\right),
$$

the map $T\left(f_{1}, \cdots, f_{n}\right)_{A}$ does not depend on the choice of a path $\gamma_{x}$.

Proof. Take another path $\gamma_{x}^{\prime}$ and set $l=\gamma_{x}^{\prime} \vee \gamma_{x}^{-1}$. By the formula (1.6.1) of [C2], we have

$$
\begin{aligned}
\int_{\gamma_{x}^{\prime}} \omega_{i_{1}} \cdots \omega_{i_{k}} & =\int_{l \gamma_{x}} \omega_{i_{1}} \cdots \omega_{i_{k}} \\
& =\sum_{0 \leq p \leq k} \int_{l} \omega_{i_{1}} \cdots \omega_{i_{p}} \int_{\gamma_{x}} \omega_{i_{p+1}} \cdots \omega_{i_{k}} \\
& =\sum_{0 \leq p \leq k} m_{i_{1} \ldots i_{k-p}}^{\prime}(l) \int_{\gamma_{x}} \omega_{i_{k-p+1}} \cdots \omega_{i_{k}}
\end{aligned}
$$


Writing simply $m_{i_{1} \ldots i_{k}}, m_{i_{1} \ldots i_{k}}^{\prime}$ for $m_{i_{1} \ldots i_{k}}(l), m_{i_{1} \ldots i_{k}}^{\prime}(l)$ respectively, we have

$$
\begin{aligned}
& A\left(\begin{array}{cccccc}
1 & \int_{\gamma_{x}^{\prime}} \omega_{1} & \int_{\gamma_{x}^{\prime}} \omega_{1} \omega_{2} & \ldots & \int_{\gamma_{x}^{\prime}} \omega_{1} \ldots \omega_{n-1} & 0 \\
& 1 & \int_{\gamma_{x}^{\prime}} \omega_{2} & \int_{\gamma_{x}^{\prime}} \omega_{2} \omega_{3} & \ldots & \int_{\gamma_{x}^{\prime}} \omega_{2} \ldots \omega_{n} \\
& & \ddots & \ddots & \ddots & \vdots \\
& & 1 & \int_{\gamma_{x}^{\prime}} \omega_{n-1} & \int_{\gamma_{x}^{\prime}} \omega_{n-1} \omega_{n} \\
& & & & 1 & \int_{\gamma_{x}^{\prime}} \omega_{n} \\
& & & & & 1
\end{array}\right) \\
& =A\left(\begin{array}{cccccc}
1 & m_{1}^{\prime} & m_{12}^{\prime} & \ldots & m_{12 \ldots n-1}^{\prime} & 0 \\
& 1 & m_{2}^{\prime} & m_{23}^{\prime} & \ldots & m_{23 \ldots n}^{\prime} \\
& & \ddots & \ddots & \ddots & \vdots \\
& & & 1 & m_{n-1}^{\prime} & m_{n-1 n}^{\prime} \\
& & & & 1 & m_{n}^{\prime} \\
& & & & & 1
\end{array}\right) \\
& \times\left(\begin{array}{cccccc}
1 & \int_{\gamma_{x}} \omega_{1} & \int_{\gamma_{x}} \omega_{1} \omega_{2} & \ldots & \int_{\gamma_{x}} \omega_{1} \ldots \omega_{n-1} & 0 \\
& 1 & \int_{\gamma_{x}} \omega_{2} & \int_{\gamma_{x}} \omega_{2} \omega_{3} & \ldots & \int_{\gamma_{x}} \omega_{2} \ldots \omega_{n} \\
& \ddots & \ddots & \ddots & \vdots \\
& & 1 & \int_{\gamma_{x}} \omega_{n-1} & \int_{\gamma_{x}} \omega_{n-1} \omega_{n} \\
& & & 1 & \int_{\gamma_{x}} \omega_{n} \\
& & & & & 1
\end{array}\right) \\
& =\left(\begin{array}{cccccc}
1 & m_{1} & m_{12} & \ldots & m_{12 \ldots n-1} & 0 \\
& 1 & m_{2} & m_{23} & \ldots & m_{23 \ldots n} \\
& & \ddots & \ddots & \ddots & \vdots \\
& & & 1 & m_{n-1} & m_{n-1 n} \\
& & & & 1 & m_{n} \\
& & & & & 1
\end{array}\right) A \\
& \times\left(\begin{array}{cccccc}
1 & \int_{\gamma_{x}} \omega_{1} & \int_{\gamma_{x}} \omega_{1} \omega_{2} & \ldots & \int_{\gamma_{x}} \omega_{1} \ldots \omega_{n-1} & 0 \\
& 1 & \int_{\gamma_{x}} \omega_{2} & \int_{\gamma_{x}} \omega_{2} \omega_{3} & \ldots & \int_{\gamma_{x}} \omega_{2} \ldots \omega_{n} \\
& \ddots & \ddots & \ddots & \vdots \\
& & 1 & \int_{\gamma_{x}} \omega_{n-1} & \int_{\gamma_{x}} \omega_{n-1} \omega_{n} \\
& & & & & \int_{\gamma_{x}} \omega_{n} \\
& & & & & 1
\end{array}\right)
\end{aligned}
$$

where the second equality follows from (3.1). By definition of $T\left(f_{1}, \ldots, f_{n}\right)_{A}$, the assumption then implies the conclusion.

Proposition 3.3. If the matrix $A$ is given by a defining system for $\left\langle f_{1}, \ldots, f_{n}\right\rangle$ in Definition 2.1, namely

$$
a_{i_{1} \ldots i_{k}}=\frac{1}{(2 \pi \sqrt{-1})^{k}} \log f_{i_{1} \ldots i_{k}}\left(x_{0}\right) \quad\left(1 \leq k \leq n-1, i_{p+1}=i_{p}+1\right),
$$


then we have

$$
m_{i_{1} \ldots i_{k}}(l) \in \mathbb{Z}\left([l] \in \pi_{1}\left(X, x_{0}\right)\right)
$$

Proof. A defining system $A$ for $\left\langle f_{1}, \ldots, f_{n}\right\rangle$ also provides a defining system, say $A$ again, for $\left\langle f_{i_{1}}, \ldots, f_{i_{k}}\right\rangle$ in an obvious manner and its value $\left\langle f_{i_{1}}, \ldots, f_{i_{k}}\right\rangle_{A}=0$ for $1 \leq k \leq n-1, i_{p+1}=i_{p}+1$. Since the holonomy of $\left\langle f_{i_{1}}, \ldots, f_{i_{k}}\right\rangle_{A}$ along $l$ is $\exp \left(2 \pi \sqrt{-1} m_{i_{1} \ldots i_{k}}(l)\right)$ by Theorem 2.5 and $(3.1)$, we have $m_{i_{1} \ldots i_{k}}(l) \in \mathbb{Z}$.

Next, we let $P:=N(\mathbb{Z}) \backslash N(\mathbb{C})$ and consider a holomorphic line bundle

$$
\pi: P \longrightarrow B
$$

induced by the natural projection. As usual, we identify $P$ with the associated principal $\mathbb{C}^{\times}$-bundle where $\exp (2 \pi \sqrt{-1} \lambda) \in \mathbb{C}^{\times}$acts on a fiber $z \in \mathbb{C} \simeq C$ by $z+\lambda$. Let $\theta$ be the 1 -form on $N(\mathbb{C})$ defined by

$$
\begin{aligned}
\theta & :=\sum_{\substack{k=0 \\
1 \leq i_{1}<i_{2}<\cdots<i_{k} \leq n-1}}^{n-1}(-1)^{k} x_{1 \ldots i_{1}} x_{i_{1}+1 \ldots i_{2}} \ldots x_{i_{k-1}+1 \ldots i_{k}} d x_{i_{k}+1 \ldots n} \\
& =\text { the }(1, n+1) \text {-component of } x^{-1} d x
\end{aligned}
$$

Proposition 3.4. The 1-form $\theta$ gives a connection form on the bundle P.

Proof. Since $(y x)^{-1} d(y x)=x^{-1} d x$ for $y \in N(\mathbb{Z}), \theta$ is left $N(\mathbb{Z})$-invariant and hence boils down to a 1 -form on $P$. To show that $\theta$ is a connection form on $P$, we need to check that (i) $\theta$ is a right $\mathbb{C}^{\times}$-invariant and (ii) $\theta$ is a Maurer-Cartan form along fibers ([KN, Ch.II,1]). (i) is, as above, obvious by the definition of $\theta$ and (ii) also follows from that $\theta$ is of the form

$$
\theta=d x_{1 \ldots n-1}+\left(\text { terms without } x_{1 \ldots n-1}\right) .
$$

Definition 3.5. Under the assumption that

$$
m_{i_{1} \ldots i_{k}}(l) \in \mathbb{Z}\left(1 \leq k \leq n-1, i_{p+1}=i_{p}+1,[l] \in \pi_{1}\left(X, x_{0}\right)\right),
$$

we define $\left\langle\left\langle f_{1}, \ldots, f_{n}\right\rangle\right\rangle_{A}$ by the isomorphism class of the pull-back of $(P, \theta)$ under $T\left(f_{1}, \ldots, f_{n}\right)_{A}$ :

$$
\left\langle\left\langle f_{1}, \ldots, f_{n}\right\rangle\right\rangle_{A}:=\text { isom. class of } T\left(f_{1}, \ldots, f_{n}\right)_{A}^{*}(P, \theta) .
$$

In the following, we shall compute the holonomy $H_{l}\left(\left\langle\left\langle f_{1}, \ldots, f_{n}\right\rangle\right\rangle_{A}\right)$ of $\left\langle\left\langle f_{1}, \ldots, f_{n}\right\rangle\right\rangle_{A}$ along $l \in \pi_{1}(X)$ and show that it coincides with $H_{l}\left(\left\langle f_{1}, \ldots, f_{n}\right\rangle_{A}\right)$.

For this, let us consider the map for a path $\gamma: I \rightarrow X$

$$
s_{\gamma}: I:=[0,1] \longrightarrow P
$$


defined by

$$
\begin{aligned}
& s_{\gamma}(t):=N(\mathbb{Z}) A Z(t),
\end{aligned}
$$

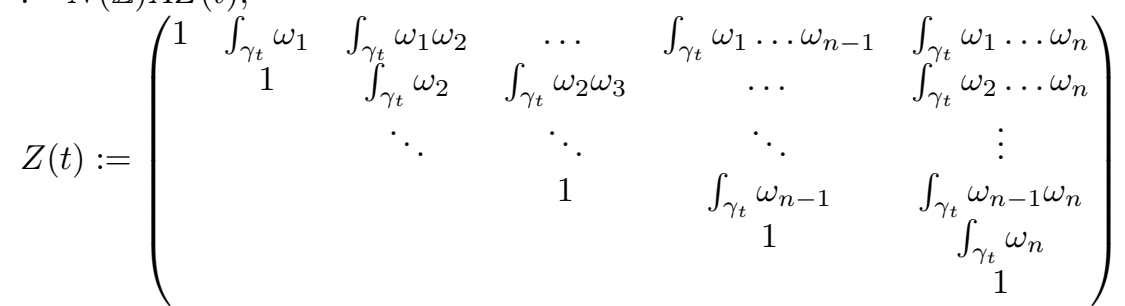

where a path $\gamma_{t}: I \rightarrow X$ is defined by $\gamma_{t}\left(t^{\prime}\right):=\gamma\left(t t^{\prime}\right)$, and we let

$$
\tilde{s}_{\gamma}: I \longrightarrow\left\langle\left\langle f_{1}, \ldots, f_{n}\right\rangle\right\rangle_{A}
$$

be the map defined by $\tilde{s}_{\gamma}(t):=\left(\gamma(t), s_{\gamma}(t)\right)$ :

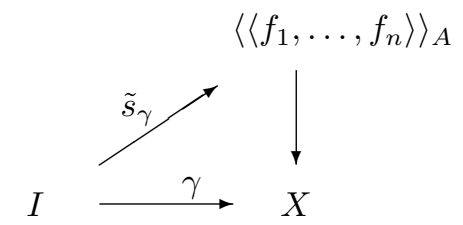

Theorem 3.6. The map $\tilde{s}_{\gamma}$ is a parallel displacement of $\gamma$ in $\left\langle\left\langle f_{1}, \ldots, f_{n}\right\rangle\right\rangle_{A}$.

Proof. Let $P X$ be the space of all paths in $X$. We regard $\int \omega_{i_{1}} \ldots \omega_{i_{k}}$ as a function on $P X$ by

$$
\left(\int \omega_{i_{1}} \ldots \omega_{i_{k}}\right)(\gamma):=\int_{\gamma} \omega_{i_{1}} \ldots \omega_{i_{k}}
$$

For $\gamma \in P X$, we define $p_{\gamma}: I \longrightarrow P X$ by

$$
p_{\gamma}(t):=\gamma_{t}
$$

Then we have

$$
\left(p_{\gamma}^{*} \int \omega_{i_{1}} \ldots \omega_{i_{k}}\right)(t)=\int_{\gamma_{t}} \omega_{i_{1}} \ldots \omega_{i_{k}} .
$$

By Proposition 1.5.2 of [C2], we have

$$
\begin{aligned}
d_{P X} \int \omega_{i_{1}} \ldots \omega_{i_{k}}= & -\sum_{p=1}^{k} \int \omega_{i_{1}} \ldots d_{X} \omega_{i_{p}} \ldots \omega_{i_{k}}-\sum_{p=1}^{k-1} \int \omega_{i_{1}} \ldots\left(\omega_{i_{p}} \wedge \omega_{i_{p+1}}\right) \ldots \omega_{i_{k}} \\
& -\operatorname{ev}_{0}^{*} \omega_{i_{1}} \wedge \int \omega_{i_{2}} \ldots \omega_{i_{k}}+\int \omega_{i_{1}} \ldots \omega_{i_{k-1}} \wedge \mathrm{ev}_{1}^{*} \omega_{i_{k}}
\end{aligned}
$$


where $\mathrm{ev}_{t}: P X \rightarrow X$ is defined by $\mathrm{ev}_{t}(\gamma):=\gamma(t)$. Hence we have

$$
\begin{aligned}
d_{I} \int_{\gamma_{t}} \omega_{i_{1}} \ldots \omega_{i_{k}} & =d_{I} p_{\gamma}^{*} \int \omega_{i_{1}} \ldots \omega_{i_{k}} \\
& =p_{\gamma}^{*} d_{P X} \int \omega_{i_{1}} \ldots \omega_{i_{k}} \\
& =p_{\gamma}^{*}\left(-\operatorname{ev}_{0}^{*} \omega_{i_{1}} \wedge \int \omega_{i_{2}} \ldots \omega_{i_{k}}+\int \omega_{i_{1}} \ldots \omega_{i_{k-1}} \wedge \mathrm{ev}_{1}^{*} \omega_{i_{k}}\right) \\
& =-\gamma_{0}^{*} \omega_{i_{1}} \wedge \int_{\gamma_{t}} \omega_{i_{2}} \ldots \omega_{i_{k}}+\int_{\gamma_{t}} \omega_{i_{1}} \ldots \omega_{i_{k-1}} \wedge \gamma_{1}^{*} \omega_{i_{k}} \\
& =\int_{\gamma_{t}} \omega_{i_{1}} \ldots \omega_{i_{k-1}} \wedge \gamma^{*} \omega_{i_{k}} .
\end{aligned}
$$

Since the connection form $\theta$ is the $(1, n+1)$-component of $x^{-1} d x(x \in N(\mathbb{C}))$ and $s_{\gamma}(t)^{-1} d_{I} s_{\gamma}(t)=(A Z(t))^{-1} d_{I}(A Z(t))=Z(t)^{-1} d_{I} Z(t)$, it suffices to show that the $(1, n+1)$-component of $Z(t)^{-1} d_{I} Z(t)=0$. In fact, we have

$$
\begin{aligned}
& \quad \begin{array}{l}
(1, n+1) \text {-entry of } Z(t) d_{I} Z(t) \\
=\sum_{k=0}^{n-1} \sum_{1 \leq i_{1}<i_{2}<\cdots<i_{k} \leq n-1}(-1)^{k} \int_{\gamma_{t}} \omega_{1} \ldots \omega_{i_{1}} \int_{g_{t}} \omega_{i_{1}+1} \ldots \omega_{i_{2}} \ldots \\
\quad \times \int_{\gamma_{t}} \omega_{i_{k-1}+1} \ldots \omega_{i_{k}} d_{I} \int_{\gamma_{t}} \omega_{i_{k}+1} \ldots \omega_{n} \\
=\sum_{k=0}^{n-1} \sum_{1 \leq i_{1}<i_{2}<\cdots<i_{k} \leq n-1}(-1)^{k} \int_{\gamma_{t}} \omega_{1} \ldots \omega_{i_{1}} \int_{g_{t}} \omega_{i_{1}+1} \ldots \omega_{i_{2}} \ldots \\
\times \int_{\gamma_{t}} \omega_{i_{k-1}+1} \ldots \omega_{i_{k}} d_{I} \int_{\gamma_{t}} \omega_{i_{k}+1} \ldots \omega_{n-1} \wedge \gamma^{*} \omega_{n}
\end{array}
\end{aligned}
$$

and here the term

$$
\begin{aligned}
& (-1)^{k} \int_{\gamma_{t}} \omega_{1} \ldots \omega_{i_{1}} \int_{g_{t}} \omega_{i_{1}+1} \ldots \omega_{i_{2}} \ldots \int_{\gamma_{t}} \omega_{i_{k-1}+1} \ldots \omega_{i_{k}} \\
& \quad \times d_{I} \int_{\gamma_{t}} \omega_{i_{k}+1} \ldots \omega_{n-1} \wedge \gamma^{*} \omega_{n}
\end{aligned}
$$

is cancelled out by the term

$$
\begin{aligned}
& (-1)^{k+1} \int_{\gamma_{t}} \omega_{1} \ldots \omega_{i_{1}} \int_{g_{t}} \omega_{i_{1}+1} \ldots \omega_{i_{2}} \ldots \int_{\gamma_{t}} \omega_{i_{k-1}+1} \ldots \omega_{i_{k}} \\
& \quad \times d_{I} \int_{\gamma_{t}} \omega_{i_{k}+1} \ldots \omega_{n-1} d_{I} \int_{\gamma_{t}} \omega_{n}
\end{aligned}
$$

and therefore the above sum $=0$.

By Theorem 3.6, we can compute the holonomy of $\left\langle\left\langle f_{1}, \ldots, f_{n}\right\rangle\right\rangle_{A}$ as follows. 
Theorem 3.7. Assume that

$$
m_{i_{1} \ldots i_{k}}(l) \in \mathbb{Z}\left(1 \leq k \leq n-1, i_{p+1}=i_{p}+1,[l] \in \pi_{1}\left(X, x_{0}\right)\right) .
$$

Then the holonomy $H_{l}\left(\left\langle\left\langle f_{1}, \ldots, f_{n}\right\rangle\right\rangle_{A}\right)$ of $\left\langle\left\langle f_{1}, \ldots, f_{n}\right\rangle\right\rangle_{A}$ along $l$ is given by

$$
H_{l}\left(\left\langle\left\langle f_{1}, \ldots, f_{n}\right\rangle\right\rangle_{A}\right)=\exp \left(2 \pi \sqrt{-1} m_{12 \ldots n}(l)\right) .
$$

Proof. The initial point of $\tilde{s}_{l}$ of $l$ based at $x_{0}$ is

$$
\left(x_{0}, s_{l}(0)=N(\mathbb{Z}) A C\right) .
$$

The terminal point of $\tilde{s}_{l}$ is $\left(x_{0}, s_{l}(1)\right)$, where

$$
\begin{aligned}
& s_{l}(1)=N(\mathbb{Z}) A\left(\begin{array}{cccccc}
1 & \int_{l} \omega_{1} & \int_{l} \omega_{1} \omega_{2} & \ldots & \int_{l} \omega_{1} \ldots \omega_{n-1} & \int_{l} \omega_{1} \ldots \omega_{n} \\
& 1 & \int_{l} \omega_{2} & \int_{l} \omega_{2} \omega_{3} & \ldots & \int_{l} \omega_{2} \ldots \omega_{n} \\
& & \ddots & \ddots & \ddots & \vdots \\
& & & 1 & \int_{l} \omega_{n-1} & \int_{l} \omega_{n-1} \omega_{n} \\
& & & & 1 & \int_{l} \omega_{n} \\
& & & & & 1
\end{array}\right) \\
& =N(\mathbb{Z}) A\left(\begin{array}{cccccc}
1 & m_{1}^{\prime} & m_{12}^{\prime} & \ldots & m_{12 \ldots n-1}^{\prime} & m_{12 \ldots n}^{\prime} \\
& 1 & m_{2}^{\prime} & m_{23}^{\prime} & \ldots & m_{23 \ldots n}^{\prime} \\
& & \ddots & \ddots & \ddots & \vdots \\
& & & 1 & m_{n-1}^{\prime} & m_{n-1 n}^{\prime} \\
& & & & 1 & m_{n}^{\prime} \\
& & & & & 1
\end{array}\right) \\
& \left.=N(\mathbb{Z})\left(\begin{array}{cccccc}
1 & m_{1} & m_{12} & \ldots & m_{12 \ldots n-1} & m_{12 \ldots n} \\
& 1 & m_{2} & m_{23} & \ldots & m_{23 \ldots n} \\
& & \ddots & \ddots & \ddots & \vdots \\
& & & 1 & m_{n-1} & m_{n-1 n} \\
& & & & 1 & m_{n} \\
& & & & & 1
\end{array}\right) A \quad \text { (by }(3.1)\right) \\
& =N(\mathbb{Z})\left(\begin{array}{cccccc}
1 & m_{1} & m_{12} & \ldots & m_{12 \ldots n-1} & 0 \\
& 1 & m_{2} & m_{23} & \ldots & m_{23 \ldots n} \\
& & \ddots & \ddots & \ddots & \vdots \\
& & & 1 & m_{n-1} & m_{n-1 n} \\
& & & & 1 & m_{n} \\
& & & & & 1
\end{array}\right) \\
& \times\left(\begin{array}{cccccc}
1 & a_{1} & a_{12} & \ldots & a_{12 \ldots n-1} & a_{12 \ldots n}+m_{12 \ldots n} \\
& 1 & a_{2} & a_{23} & \ldots & a_{23 \ldots n} \\
& & \ddots & \ddots & \ddots & \vdots \\
& & & 1 & a_{n-1} & a_{n-1 n} \\
& & & & 1 & a_{n} \\
& & & & & 1
\end{array}\right)
\end{aligned}
$$




$$
=N(\mathbb{Z})\left(\begin{array}{cccccc}
1 & a_{1} & a_{12} & \ldots & a_{12 \ldots n-1} & a_{12 \ldots n}+m_{12 \ldots n} \\
& 1 & a_{2} & a_{23} & \ldots & a_{23 \ldots n} \\
& & \ddots & \ddots & \ddots & \vdots \\
& & & 1 & a_{n-1} & a_{n-1 n} \\
& & & & 1 & a_{n} \\
& & & & & 1
\end{array}\right) \quad \text { (by assumption) }
$$

where we write simply $m_{i_{1} \ldots i_{k}}, m_{i_{1} \ldots i_{k}}^{\prime}$ for $m_{i_{1} \ldots i_{k}}(l), m_{i_{1} \ldots i_{k}}^{\prime}(l)$ respectively.

Hence the holonomy $H_{l}\left(\left\langle\left\langle f_{1}, \ldots, f_{n}\right\rangle\right\rangle\right)$ is given by $\exp \left(2 \pi \sqrt{-1} m_{12 \ldots n}\right)$.

Theorem 3.8. If the matrix $A$ is given by a defining system for $\left\langle f_{1}, \ldots, f_{n}\right\rangle$ in Definition 2.1, namely

$$
a_{i_{1} \ldots i_{k}}=\frac{1}{(2 \pi \sqrt{-1})^{k}} \log f_{i_{1} \ldots i_{k}}\left(x_{0}\right) \quad\left(1 \leq k \leq n-1, i_{p+1}=i_{p}+1\right),
$$

then we have

$$
\left\langle f_{1}, \ldots, f_{n}\right\rangle_{A}=\left\langle\left\langle f_{1}, \ldots, f_{n}\right\rangle\right\rangle_{A} .
$$

Proof. By Proposition 3.3, the assumption of Theorem 3.7 is satisfied and hence $H_{l}\left(\left\langle\left\langle f_{1}, \ldots, f_{n}\right\rangle\right\rangle_{A}\right)=\exp \left(2 \pi \sqrt{-1} m_{12 \ldots n}(l)\right)$. Since $M_{12 \ldots n}(l)=(2 \pi \sqrt{-1})^{n} m_{12 \ldots n}(l)$ by Theorem 2.5 and $(3.1)$, we have $H_{l}\left(\left\langle f_{1}, \ldots, f_{n}\right\rangle_{A}\right)=H_{l}\left(\left\langle\left\langle f_{1}, \ldots, f_{n}\right\rangle\right\rangle_{A}\right)$ for all $[l] \in \pi_{1}\left(X, x_{0}\right)$. Since the isomorphism class of line bundles with holomorphic connection on $X$ is determined by its holonomy representation (1.3), $\left\langle f_{1}, \cdots, f_{n}\right\rangle_{A}$ coincides with $\left\langle\left\langle f_{1}, \cdots, f_{n}\right\rangle\right\rangle_{A}$.

\section{Properties of polysymbols}

In this section, using our holonomy formula, Theorem 2.5, we show some basic properties of polysymbols which generalize those of the classical tame symbol. We keep the same notations as in Sections 2 and 3.

Proposition 4.1 (multiplicativity). Assume $f_{j}=f_{j}^{\prime} \cdot f_{j}^{\prime \prime}$ for meromorphic functions $f_{j}^{\prime}, f_{j}^{\prime \prime}$ on $\bar{X}$. Suppose that $A^{\prime}=\left\{\left(q_{i_{1} \ldots i_{k}}^{\prime}, \log f_{i_{1} \ldots i_{k}}^{\prime}\right)\right\}$ and $A^{\prime \prime}=\left\{\left(q_{i_{1} \ldots i_{k}}^{\prime \prime}, f_{i_{1} \ldots i_{k}}^{\prime \prime}\right)\right\}$ are defining systems for $\left\langle f_{1}, \ldots, f_{j}^{\prime}, \ldots, f_{n}\right\rangle$ and $\left\langle f_{1}, \ldots, f_{j}^{\prime \prime}, \ldots f_{n}\right\rangle$ respectively as in Definition 2.1 such that $f_{i_{1} \ldots i_{k}}^{\prime}=f_{i_{1} \ldots i_{k}}^{\prime \prime}$ if $j \notin\left\{i_{1}, \ldots, i_{k}\right\}$. Then an array $A=$ $\left\{\left(q_{i_{1} \ldots i_{k}}, \log f_{i_{1} \ldots i_{k}}\right)\right\}$ defined by

$$
\begin{aligned}
& q_{i_{1} \ldots i_{k}}:= \begin{cases}q_{i_{1} \ldots i_{k}}^{\prime}=q_{i_{1} \ldots i_{k}}^{\prime \prime} & j \notin\left\{i_{1}, \ldots, i_{k}\right\}, \\
q_{i_{1} \ldots i_{k}}^{\prime} \cdot q_{i_{1} \ldots i_{k}}^{\prime \prime}, & j \in\left\{i_{1}, \ldots, i_{k}\right\}\end{cases} \\
& f_{i_{1} \ldots i_{k}}:= \begin{cases}f_{i_{1} \ldots i_{k}}^{\prime}=f_{i_{1} \ldots i_{k}}^{\prime \prime} & j \notin\left\{i_{1}, \ldots, i_{k}\right\}, \\
f_{i_{1} \ldots i_{k}}^{\prime} \cdot f_{i_{1} \ldots i_{k}}^{\prime \prime}, & j \in\left\{i_{1}, \ldots, i_{k}\right\}\end{cases}
\end{aligned}
$$

gives a defining system for $\left\langle f_{1}, \ldots, f_{j}^{\prime} f_{j}^{\prime \prime}, \ldots, f_{n}\right\rangle$ and we have

$$
\left\langle f_{1}, \ldots, f_{j}^{\prime} f_{j}^{\prime \prime}, \ldots, f_{n}\right\rangle_{A}=\left\langle f_{1}, \ldots, f_{j}^{\prime}, \ldots, f_{n}\right\rangle_{A^{\prime}}+\left\langle f_{1}, \ldots, f_{j}^{\prime \prime}, \ldots, f_{n}\right\rangle_{A^{\prime \prime}} .
$$

Proof. It is easy to see that $A$ is a defining system for $\left\langle f_{1}, \ldots, f_{j}^{\prime} f_{j}^{\prime \prime}, \ldots, f_{n}\right\rangle$ under 
the assumption. By Theorem 2.5 and by the general formulas

$$
\log (f g)\left(x_{0}\right)=\log f\left(x_{0}\right)+\log g\left(x_{0}\right), \frac{d(f g)}{f g}=\frac{d f}{f}+\frac{d g}{g},
$$

we have

$$
H_{l}\left(\left\langle f_{1}, \ldots, f_{j}^{\prime} f_{j}^{\prime \prime}, \ldots, f_{n}\right\rangle_{A}\right)=H_{l}\left(\left\langle f_{1}, \ldots, f_{j}^{\prime}, \ldots, f_{n}\right\rangle_{A^{\prime}}\right) \cdot H_{l}\left(\left\langle f_{1}, \ldots, f_{j}^{\prime \prime}, \ldots, f_{n}\right\rangle_{A^{\prime \prime}}\right)
$$

for any $[l] \in \pi_{1}\left(X, x_{0}\right)$. This proves the assertion.

Proposition 4.2 (symmetric relation). Let $\mathfrak{S}_{n}$ be the symmetric group on $\{1, \ldots, n\}$. For each permutation $\sigma \in \mathfrak{S}_{n}$, let $\sigma(A)=\left\{\left(q_{\sigma\left(i_{1}\right) \ldots \sigma\left(i_{k}\right)}, f_{\sigma\left(i_{1}\right) \ldots \sigma\left(i_{k}\right)}\right)\right\}$ be a defining system for $\left\langle f_{\sigma(1)}, \ldots, f_{\sigma(n)}\right\rangle$. Then we have

$$
\sum_{\sigma \in \mathfrak{S}_{n}}\left\langle f_{\sigma(1)}, \ldots, f_{\sigma(n)}\right\rangle_{\sigma(A)}=0
$$

Proof. By Theorem 2.5 and cancellation in pairs, we have

$$
\prod_{\sigma \in \mathfrak{S}_{n}} H_{l}\left(\left\langle f_{\sigma(1)}, \ldots, f_{\sigma(n)}\right\rangle_{\sigma(A)}\right)=\exp \left(\frac{1}{(2 \pi \sqrt{-1})^{n-1}} \sum_{\sigma \in \mathfrak{S}_{n}} \int_{l} \frac{d f_{\sigma(1)}}{f_{\sigma(1)}} \cdots \frac{d f_{\sigma(n)}}{f_{\sigma(n)}}\right)
$$

for any $[l] \in \pi_{1}\left(X, x_{0}\right)$. Using the general formula (1.5.1) of [C1]

$$
\int_{l} w_{1} \cdots w_{r} \int_{l} w_{r+1} \cdots w_{r+s}=\sum_{\sigma \in S H} \int_{l} w_{\sigma(1)} \cdots w_{\sigma(r+s)}
$$

where $S H$ denotes the the set of all $(r, s)$-shuffles, i.e. permutations $\sigma$ with $\sigma^{-1}(1)<$ $\cdots<\sigma^{-1}(r), \sigma^{-1}(r+1)<\cdots<\sigma^{-1}(r+s)$, we have

$$
\sum_{\sigma \in \mathfrak{S}_{n}} \int_{l} \frac{d f_{\sigma(1)}}{f_{\sigma(1)}} \cdots \frac{d f_{\sigma(n)}}{f_{\sigma(n)}}=\int_{l} \frac{d f_{1}}{f_{1}} \cdots \int_{l} \frac{d f_{n}}{f_{n}} \in(2 \pi \sqrt{-1})^{n} \mathbb{Z} .
$$

Hence we have

$$
\prod_{\sigma \in \mathfrak{S}_{n}} H_{l}\left(\left\langle f_{\sigma(1)}, \ldots, f_{\sigma(n)}\right\rangle_{\sigma(A)}\right)=1
$$

for any $[l] \in \pi_{1}\left(X, x_{0}\right)$. This proves the assertion.

The following theorem is regarded as a generalization of the classical reciprocity law of Tate and Weil (Ch.III,4 of [Se]).

Theorem 4.3 (reciprocity law). Assume that $\left\langle f_{1}, \ldots, f_{n}\right\rangle$ is defined. Then we have the following product formula

$$
\prod_{x \in \bar{X}}\left\{f_{1}, \ldots, f_{n}\right\}_{x}=1
$$

Proof. Let $Y$ be the surface obtained by removing from $\bar{X}$ small open disks centered 
at points in $\cup_{i=1}^{n} \operatorname{supp}\left(f_{i}\right)$ and let $\partial Y=l_{1} \cup \cdots \cup l_{N}$ (disjoint union) be the boundary of $Y$. Then for any defining system $A$ for $\left\langle f_{1}, \ldots, f_{n}\right\rangle$, we have

$$
\begin{aligned}
\prod_{x \in \bar{X}} & H_{l_{x}}\left(\left\langle f_{1}, \ldots, f_{n}\right\rangle_{A}\right) \\
& =\prod_{i=1}^{N} H_{l_{i}}\left(\left\langle f_{1}, \ldots, f_{n}\right\rangle_{A}\right) \\
& =\exp \left(\int_{\operatorname{Int}(Y)}-\text { curv. of }\left\langle f_{1}, \ldots, f_{n}\right\rangle_{A}\right)([\mathrm{Br}, \operatorname{Prop} .2 .4 .6,6.1 .1]) \\
& =1,
\end{aligned}
$$

since the curvature of $\left\langle f_{1}, \ldots, f_{n}\right\rangle_{A}$ is zero. By Definition 2.3, the assertion is proved.

\section{Variation of mixed Hodge structure}

In this section, we show that trivializations of polysymbols give variations of mixed Hodge structure (cf. Section 7 of $[\mathrm{H}]$ ).

First, recall that a variation of mixed Hodge structure on a complex manifold $X$ consists of a triple $\left(V_{\mathbb{Z}}, W_{\bullet}, F^{\bullet}\right)$

(i) a local system $V_{\mathbb{Z}}$ of finitely generated $\mathbb{Z}$-modules on $X$;

(ii) an increasing filtration $W_{\bullet}$ of $V_{\mathbb{Z}}$ by local systems of finitely generated

$\mathbb{Z}$-modules;

(iii) a decreasing filtration $F^{\bullet}$ of $V_{\mathbb{Z}} \otimes \mathcal{O}_{X}$ by holomorphic subbundles which are required to satisfy

(1) (Griffiths' transversality) $\nabla F^{i} \subset \Omega^{1} \otimes F^{i-1}$

where $\nabla$ is the canonical flat connection on $V_{\mathbb{Z}} \otimes \mathcal{O}_{X}$;

(2) for each point in $X, W \bullet$ and $F^{\bullet}$ define a mixed Hodge structure on each fiber.

Now, let us go back to our previous setting and keep the same notations as in Section 2,3 . So, $f_{1}, \ldots, f_{n}$ are meromorphic functions on a closed Riemann surface $\bar{X}$ and $X=\bar{X} \backslash \cup_{i=1}^{n} S\left(f_{i}\right)$.

Definition 5.1. A trivialization of a polysymbol $\left\langle f_{1}, \ldots, f_{n}\right\rangle_{A}$ relative to a defining system $A=\left\{\alpha_{i_{1} \ldots i_{k}}\right\}=\left\{\left(q_{i_{1} \ldots i_{k}}, \log f_{i_{1} \ldots i_{k}}\right)\right\}$ is a 1-cochain $\alpha_{1 \ldots n}=\left(q_{1 \ldots n}, \log f_{1 \ldots n}\right)$ satisfying the relation

$$
d \alpha_{1 \ldots n}=\alpha_{1 \ldots n-1} \cup \alpha_{n}+\cdots+\alpha_{1} \cup \alpha_{2 \ldots n} .
$$

Assume in the following that we have a trivialization of $\left\langle f_{1}, \ldots, f_{n}\right\rangle_{A}$ as in Definition 5.1 , which yields $\left\langle f_{1}, \ldots, f_{n}\right\rangle_{A}=0$. We set

$$
a_{i_{1} \ldots i_{k}}=\frac{1}{(2 \pi \sqrt{-1})^{k}} \log f_{i_{1} \ldots i_{k}}\left(x_{0}\right) .
$$


For the standard basis $\left\{e_{0}, \ldots, e_{n}\right\}$ of $\mathbb{C}^{n+1}$, we consider the vectors $v_{0}, \ldots, v_{n}$ defined by

$$
\left(\begin{array}{c}
v_{0} \\
v_{1} \\
\vdots \\
v_{n}
\end{array}\right)=\left(\begin{array}{cccc}
1 & a_{1} & \ldots & a_{1 \ldots n} \\
& \ddots & \ddots & \vdots \\
& & 1 & a_{n} \\
& & & 1
\end{array}\right)\left(\begin{array}{cccc}
1 & \int_{\gamma_{x}} \omega_{1} & \ldots & \int_{\gamma_{x}} \omega_{1} \ldots \omega_{n} \\
& \ddots & \ddots & \vdots \\
& & 1 & \int_{\gamma_{x}} \omega_{n} \\
& & & 1
\end{array}\right)\left(\begin{array}{c}
e_{0} \\
(2 \pi \sqrt{-1}) e_{1} \\
\vdots \\
(2 \pi \sqrt{-1})^{n} e_{n} .
\end{array}\right)
$$

The proof of Lemma 3.2 shows that the map $F: X \rightarrow N(\mathbb{C})$ defined by

$$
F(x)=\left(\begin{array}{cccc}
1 & a_{1} & \ldots & a_{1 \ldots n} \\
& \ddots & \ddots & \vdots \\
& & 1 & a_{n} \\
& & & 1
\end{array}\right)\left(\begin{array}{cccc}
1 & \int_{\gamma_{x}} \omega_{1} & \ldots & \int_{\gamma_{x}} \omega_{1} \ldots \omega_{n} \\
& \ddots & \ddots & \vdots \\
& & 1 & \int_{\gamma_{x}} \omega_{n} \\
& & & 1
\end{array}\right)
$$

modulo $N(\mathbb{Z})$ does not depend on the choice of a path $\gamma_{x}$. Therefore, the $\mathbb{Z}$-span $V_{\mathbb{Z}}(x)$ of the vectors $v_{0}, \ldots, v_{n}$ is well-defined. These vectors induce an increasing filtration of $V_{\mathbb{Z}}(x)$ defined by

$$
W_{0}=\operatorname{span}_{\mathbb{Z}}\left\{v_{0}, \ldots, v_{n}\right\}, W_{-1}=\operatorname{span}_{\mathbb{Z}}\left\{v_{1}, \ldots, v_{n}\right\}, \ldots, W_{-n}=\operatorname{span}_{\mathbb{Z}}\left\{v_{n}\right\} .
$$

In addition, we have a decreasing filtration on $\mathbb{C}^{n+1}$ defined by

$$
F^{0}=\operatorname{span}_{\mathbb{C}}\left\{e_{0}\right\}, F^{-1}=\operatorname{span}_{\mathbb{C}}\left\{e_{0}, e_{1}\right\}, \ldots, F^{-n}=\operatorname{span}_{\mathbb{C}}\left\{e_{0}, \ldots e_{n}\right\} .
$$

Theorem 5.2. The triple $\left(V_{\mathbb{Z}}, W_{\bullet}, F^{\bullet}\right)$ defined as above is a variation of mixed Hodge structure on $X$ with $V_{\mathbb{Z}} \otimes \mathcal{O}_{X}=X \times \mathbb{C}^{n+1}$ whose graded quotients of $W \bullet$ are $\mathbb{Z}(0), \mathbb{Z}(1), \ldots, \mathbb{Z}(n)$.

Proof. First, we consider a connection $\nabla$ on $X \times \mathbb{C}^{n+1} \rightarrow X$ defined by

$$
\nabla v=d v-v \omega
$$

for a section $v: X \rightarrow \mathbb{C}^{n+1}$, where

$$
\omega=2 \pi \sqrt{-1}\left(\begin{array}{cccc}
0 & \omega_{1} & \cdots & 0 \\
& \ddots & \ddots & \vdots \\
& & 0 & \omega_{n} \\
& & & 0
\end{array}\right)
$$

The connection $\nabla$ is flat because

$$
d \omega=0 \text { and } \omega \wedge \omega=0
$$

by the fact that each component of $\omega$ is a closed and holomorphic 1-form on a 1dimensional complex manifold $X$. By the definition of the (multi-valued) map $F$ : $X \rightarrow N(\mathbb{C})$, we find that the vectors $v_{1}, \ldots, v_{n}$ as sections satisfy $\nabla v_{i}=0$. Therefore, $W_{0}, W_{-1}, \ldots, W_{-n}$ are local systems on $X$ because the monodromy representation has values in $N(\mathbb{Z})$. The Griffiths' transversality follows from the fact that the connection matrix $\omega$ is a strictly upper triangular matrix.

This theorem means that polysymbols are obstructions to getting variations of mixed Hodge structure. 
Example 5.3. Let $\bar{X}=\mathbb{P}^{1}=\mathbb{C} \cup\{\infty\}$ and consider invertible holomorphic functions*

$$
f_{1}=1-z, f_{2}=z, f_{3}=(\zeta-z)^{6}
$$

on $X=\mathbb{P}^{1} \backslash\{0,1, \zeta, \infty\}$ where $\zeta:=\exp \left(\frac{2 \pi \sqrt{-1}}{6}\right)$ and $z$ is a coordinate in $\mathbb{C}$.

Proposition 5.4. $\left\langle f_{i}, f_{j}\right\rangle=0(1 \leq i \neq j \leq 3)$.

Proof. It suffices to show $\left\langle 1-z,(\zeta-z)^{6}\right\rangle=\left\langle z,(\zeta-z)^{6}\right\rangle=0$. Recall that if $f, 1-f \in$ $H^{0}\left(X, \mathcal{O}_{X}^{\times}\right)$, then $\langle f, 1-f\rangle=0$ (Cor. 1.15 of [Bl1]). Then using $\zeta^{6}=1$, we have

$$
\begin{aligned}
\left\langle z,(\zeta-z)^{6}\right\rangle & =6\langle z, \zeta-z\rangle \\
& =6\langle z, \zeta\rangle+6\left\langle z, 1-\zeta^{-1} z\right\rangle \\
& =6\left\langle z, 1-\zeta^{-1} z\right\rangle \\
& =6\left\langle\zeta^{-1} z, 1-\zeta^{-1} z\right\rangle \\
& =0
\end{aligned}
$$

Noting $(\zeta-1)^{6}=1$ and changing $z$ by $1-z,\left\langle 1-z,(\zeta-z)^{6}\right\rangle=0$ is proved.

Let $L i_{2}(z)$ be the dilogarithm function defined by

$$
L i_{2}(z):=-\int_{0}^{z} \log (1-z) \frac{d z}{z}=\sum_{n=1}^{\infty} \frac{z^{n}}{n^{2}}
$$

Proposition 5.5. The functions

$$
\begin{aligned}
f_{12} & :=\exp \left(\int_{\zeta}^{z} \log (1-z) \frac{d z}{z}\right)=\exp \left(-L i_{2}(z)+L i_{2}(\zeta)\right), \\
f_{23}(z) & :=\exp \left(-\int_{1}^{z} \log (z) \frac{d z}{\zeta-z}\right)
\end{aligned}
$$

give rise to a defining system for $\left\langle 1-z, z,(\zeta-z)^{6}\right\rangle$ so that the line bundle with holomorphic connection $\left\langle 1-z, z,(\zeta-z)^{6}\right\rangle_{A}$ is trivial.

Proof. It suffices to show that $\left\langle 1-z, z,(\zeta-z)^{6}\right\rangle_{A}$ has a flat section over $X$. We define a (possibly multi-valued) map $\tilde{t}: X \rightarrow N(\mathbb{Z}) \backslash N(\mathbb{C})$ by

$$
\tilde{t}(z):=N(\mathbb{Z}) t(z), t(z):=\left(\begin{array}{cccc}
1 & t_{1}(z) & t_{12}(z) & t_{123}(z) \\
0 & 1 & t_{2}(z) & t_{23}(z) \\
0 & 0 & 1 & t_{3}(z) \\
0 & 0 & 0 & 1
\end{array}\right),
$$

${ }^{*}$ This example and Proposition 5.4 are due to M. Asakura. 
where

$$
\begin{aligned}
t_{1}(z) & :=\frac{1}{2 \pi \sqrt{-1}} \log (1-z), t_{2}(z):=\frac{1}{2 \pi \sqrt{-1}} \log (z), \\
t_{3}(z) & :=\frac{6}{2 \pi \sqrt{-1}}(\log (\zeta-z)-\log (\zeta-1)) \\
t_{12}(z) & :=\frac{1}{(2 \pi \sqrt{-1})^{2}}\left(-L i_{2}(z)+L i_{2}(\zeta)\right), t_{23}(z):=\frac{-6}{(2 \pi \sqrt{-1})^{2}} \int_{1}^{z} \log (z) \frac{d z}{\zeta-z} \\
t_{123}(z) & :=\frac{6}{(2 \pi \sqrt{-1})^{3}}\left(\int_{0}^{z} L i_{2}(z) \frac{d z}{\zeta-z}-L i_{2}(\zeta) \log (\zeta-z)\right) .
\end{aligned}
$$

The monodromies around 0 of $t_{2}(z), t_{23}(z)$ and other $t_{*}(z)$ 's are $1, t_{3}(z)$ and 0 respectively and so the analylic continuation of $t(z)$ around 0 is

$$
\begin{aligned}
\left(\begin{array}{cccc}
1 & t_{1}(z) & t_{12}(z) & t_{123}(z) \\
0 & 1 & t_{2}(z)+1 & t_{23}(z)+t_{3}(z) \\
0 & 0 & 1 & t_{3}(z) \\
0 & 0 & 0 & 1
\end{array}\right) & =\left(\begin{array}{cccc}
1 & 0 & 0 & 0 \\
0 & 1 & 1 & 0 \\
0 & 0 & 1 & 0 \\
0 & 0 & 0 & 1
\end{array}\right) t(z) \\
& \equiv t(z) \bmod N(\mathbb{Z})
\end{aligned}
$$

The monodromies around 1 of $t_{1}(z), t_{12}(z), t_{123}(z)$ and other $t_{*}(z)$ 's are $1, t_{2}(z), t_{23}(z)$ and 0 respectively and so the analytic continuation of $t(z)$ around 1 is

$$
\begin{aligned}
\left(\begin{array}{cccc}
1 & t_{1}(z)+1 & t_{12}(z)+t_{2}(z) & t_{123}(z)+t_{23}(z) \\
0 & 1 & t_{2}(z) & t_{23}(z) \\
0 & 0 & 1 & t_{3}(z) \\
0 & 0 & 0 & 1
\end{array}\right) & =\left(\begin{array}{cccc}
1 & 1 & 0 & 0 \\
0 & 1 & 0 & 0 \\
0 & 0 & 1 & 0 \\
0 & 0 & 0 & 1
\end{array}\right) t(z) \\
& \equiv t(z) \bmod N(\mathbb{Z}) .
\end{aligned}
$$

The monodromies around $\zeta$ of $t_{3}(z), t_{23}(z)$ and other $t_{*}(z)$ 's are $6,-1$ and 0 respectively and so the analytic continuation of $t(z)$ around $\zeta$ is

$$
\begin{aligned}
\left(\begin{array}{cccc}
1 & t_{1}(z) & t_{12}(z) & t_{123}(z) \\
0 & 1 & t_{2}(z) & t_{23}(z)-1 \\
0 & 0 & 1 & t_{3}(z)+6 \\
0 & 0 & 0 & 1
\end{array}\right) & =\left(\begin{array}{cccc}
1 & 0 & 0 & 0 \\
0 & 1 & 0 & -1 \\
0 & 0 & 1 & 6 \\
0 & 0 & 0 & 1
\end{array}\right) t(z) \\
& \equiv t(z) \bmod N(\mathbb{Z})
\end{aligned}
$$

Hence the map $\tilde{t}: X \rightarrow N(\mathbb{Z}) \backslash N(\mathbb{C})$ is a single valued map. Moreover, by a straightforward computation, we have

$$
\tilde{t}^{*}(\theta)=0 .
$$

Therefore the map $\tilde{t}$ gives a flat section of $\left\langle 1-z, z,(\zeta-z)^{6}\right\rangle_{A}$ over $X$.

Acknowledgement. We would like to thank M. Kapranov for suggesting the problem, how one can define a triple resultant, which was a motivation of our work. We are thankful to Y. Takeda for useful discussions and to C. Deninger for informing us of the recent papers $[\mathrm{Sc}]$ and $[\mathrm{W}]$ as well as $[\mathrm{Dn}]$. Thanks are also due to the referee for useful remarks. We are very grateful to M. Asakura for telling us an interesting 
example. The authors are supported in part by the Grants-in-Aid for Scientific Research, Japan Society for the Promotion of Science.

\section{References}

[Bl] S. Bloch, The dilogarithm and extensions of Lie algebras, Algebraic K-theory, Evanston 1980 (Proc. Conf., Northwestern Univ., Evanston, Ill., 1980), pp. 1-23, Lecture Notes in Math., 854, Springer, Berlin-New York, 1981.

[Br] J. L. Brylinski, Loop spaces, characteristic classes and geometric quantization, Progress in Mathematics, 107, Birkhäuser Boston, Inc., Boston, MA, 1993.

[C1] K. T. Chen, Algebras of iterated path integrals and fundamental groups, Trans. Amer. Math. Soc. 156 (1971), 359-379.

[C2] _ Iterated path integrals, Bull. Amer. Math. Soc. 83 (1977), no. 5, 831-879.

[Dl] P. Deligne, Le symbole modéré, Inst. Hautes Etudes Sci. Publ. Math. 73, (1991), 147-181.

[Dn] C. Deninger, Higher order operations in Deligne cohomology, Invent. Math. 120 (1995), no. 2, 289-315.

[EV] H. Esnault and E. Viehweg, Deligne-Beilinson cohomology, Beilinson's conjectures on special values of L-functions, 43-91, Perspect. Math., 4, Academic Press, Boston, MA, 1988.

[H] R. Hain, Classical polylogarithms, Motives (Seattle, WA, 1991), 3-42, Proc. Sympos. Pure Math., 55, Part 2, Amer. Math. Soc., Providence, RI, 1994.

[KN] S. Kobayashi and K. Nomizu, Foundations of differential geometry, Vol I. Interscience Publishers, a division of John Wiley \& Sons, New York-London 1963.

[Kr] D. Kraines, Massey higher products, Trans. Amer. Math. Soc. 124, (1966), 431-449.

[Ms] W. S. Massey, Higher order linking numbers, J. Knot Theory Ramifications 7 (1998), no. 3, 393-414.

[My] J. P. May, Matric Massey products, J. Algebra 12 (1969), 533-568.

[Mo] M. Morishita, Analogies between knots and primes, 3-manifolds and number rings, to appear in Sugaku Expositions, Amer. Math. Soc. (Analogies between prime numbers and knots, (Japanese) Sugaku 58, (2006), no. 1, 40-63.)

[R] D. Ramakrishnan, A regulator for curves via the Heisenberg group, Bull. Amer. Math. Soc. (N.S.) 5 (1981), no. 2, 191-195.

[Sc] A Schwarzhaupt, Massey products in Deligne Beilinson cohomology, preprint.

[Se] J. P. Serre, Groupes algébriques et corps de classes, Publications de l'institut de mathématique de l'université de Nancago, VII. Hermann, Paris 1959.

[Tu] V.G. Turaev, Milnor's invariants and Massey products, English transl. J. Soviet Math. 12, (1979), 128-137.

[W] T. Wenger, Massey Products in Deligne Cohomology, preprint.

Graduate School of Mathematics, Kyushu University, 6-10-1, Hakozaki, Higashi-ku, FUKUOKA, 812-8581 JAPAN

E-mail address: morisita@math.kyushu-u.ac.jp

Department of Mathematics, Tokyo Institute of Technology, 2-12-1 Oh-Okayama,

Meguro-KU, TOKYO 152-8551, JAPAN

E-mail address: tera@math.titech.ac.jp 